\title{
Culture of pulmonary artery endothelial cells from pulmonary artery catheter balloon tips: considerations for use in pulmonary vascular disease
}

\author{
Corey E. Ventetuolo ${ }^{1,2}$, Jason M. Aliotta ${ }^{1}$, Julie Braza ${ }^{3}$, Havovi Chichger ${ }^{4}$, \\ Mark Dooner ${ }^{5}$, Donald McGuirl ${ }^{6}$, Christopher J. Mullin ${ }^{1}$, Julie Newton ${ }^{3}$, \\ Mandy Pereira ${ }^{5}$, Amy Princiotto $^{3}$, Peter J. Quesenberry ${ }^{1}$, Thomas Walsh ${ }^{5}{ }^{5}$, \\ Mary Whittenhall ${ }^{1}$, James R. Klinger ${ }^{1}$ and Elizabeth 0 . Harrington ${ }^{1,3}$
}

\begin{abstract}
Affiliations: ${ }^{1}$ Dept of Medicine, Brown University, Providence, RI, USA. ${ }^{2}$ Dept of Health Services, Policy and Practice, Brown University, Providence, RI, USA. ${ }^{3}$ Vascular Research Laboratory, Providence Veterans Affairs Medical Center, Providence, RI, USA. ${ }^{4}$ Biomedical Research Group, Dept of Biomedical and Forensic Sciences, Anglia Ruskin University, Cambridge, UK. ${ }^{5}$ Lifespan Hospital System, Providence, RI, USA. ${ }^{6}$ Tufts University School of Medicine, Boston, MA, USA.
\end{abstract}

Correspondence: Corey E. Ventetuolo, Division of Pulmonary, Critical Care and Sleep Medicine, Rhode Island Hospital, 593 Eddy Street, POB Suite 224, Providence, RI 02903, USA. E-mail: corey ventetuolodabrown.edu

@ERSpublications

Pulmonary artery endothelial cells (PAECs) from pulmonary artery catheter balloons used during routine right heart catheterisation can be cultured and sustained to study endothelial cell dysfunction at various stages of pulmonary hypertension http://bit.ly/2RL6dTc

Cite this article as: Ventetuolo CE, Aliotta JM, Braza J, et al. Culture of pulmonary artery endothelial cells from pulmonary artery catheter balloon tips: considerations for use in pulmonary vascular disease. Eur Respir J 2020; 55: 1901313 [https://doi.org/10.1183/13993003.01313-2019].

ABSTRACT Endothelial dysfunction is a hallmark of pulmonary arterial hypertension (PAH) but there are no established methods to study pulmonary artery endothelial cells (PAECs) from living patients. We sought to culture PAECs from pulmonary artery catheter (PAC) balloons used during right-heart catheterisation (RHC) to characterise successful culture attempts and to describe PAEC behaviour.

PAECs were grown in primary culture to confluence and endothelial cell phenotype was confirmed. Standard assays for apoptosis, migration and tube formation were performed between passages three to eight. We collected 49 PAC tips from 45 subjects with successful PAEC culture from 19 balloons (39\%).

There were no differences in subject demographic details or RHC procedural details in successful versus unsuccessful attempts. However, for subjects who met haemodynamic criteria for $\mathrm{PAH}$, there was a higher but nonsignificant $(\mathrm{p}=0.10)$ proportion amongst successful attempts ( 10 out of $19,53 \%)$ versus unsuccessful attempts (nine out of $30,30 \%)$. A successful culture was more likely in subjects with a lower cardiac index $(\mathrm{p}=0.03)$ and higher pulmonary vascular resistance $(\mathrm{p}=0.04)$. PAECs from a subject with idiopathic PAH were apoptosis resistant compared to commercial PAECs $(\mathrm{p}=0.04)$ and had reduced migration compared to PAECs from a subject with portopulmonary hypertension with high cardiac output $(\mathrm{p}=0.01)$. PAECs from a subject with HIVassociated PAH formed fewer $(\mathrm{p}=0.01)$ and shorter $(\mathrm{p}=0.02)$ vessel networks compared to commercial PAECs.

Sustained culture and characterisation of PAECs from RHC balloons is feasible, especially in PAH with high haemodynamic burden. This technique may provide insight into endothelial dysfunction during $\mathrm{PAH}$ pathogenesis.

This article has an editorial commentary: https://doi.org/10.1183/13993003.00122-2020

This article has supplementary material available from erj.ersjournals.com

Received: 3 July 2019 | Accepted after revision: 10 Dec 2019

Copyright @ERS 2020 


\section{Introduction}

Pulmonary arterial hypertension (PAH) is a progressive disease of the pulmonary circulation characterised by endothelial dysfunction. Aberrant pulmonary artery endothelial cells (PAECs) are a hallmark of disease pathogenesis [1-3], but controversy exists about whether cellular proliferation or apoptosis dominate in the pulmonary vasculature. The balance between neo-angiogenesis and vascular rarefaction may shift over a patient's disease course [4]. Current methodologies to study human PAECs include harvested cells from the explanted or post-mortem lungs of PAH patients, commercially available non-diseased PAECs and the differentiation of endothelial cells from peripheral blood or dermal biopsies [5-7]. While these methods have strengths, cells are sourced from end-stage PAH patients, non-diseased humans, or outside of the pulmonary vasculature. There is a critical need for a reliable method to culture and expand PAECs from living patients to examine changes in endothelial cell function both over time and in response to current and investigational PAH therapies.

Right-heart catheterisation (RHC) is the fundamental diagnostic procedure in pulmonary vascular disease evaluation and is often repeated throughout a PAH patient's disease course to guide therapeutic decisions. This procedure could provide a source of primary cells to be propagated ex vivo and an opportunity to collect PAECs across repetitive samples from patients at variable stages of disease. A single report has described the successful collection of cells with endothelial cell phenotype from pulmonary artery catheter (PAC) balloons in 24 patients with PAH for as long as 20 days in culture; however, it was unclear if this method could result in enough cells to perform functional experiments without phenotypic transformation [8]. We set out to advance this methodology and to determine 1) the features of subjects and procedures in which this technique most often yielded robust numbers of viable PAECs, 2) the most successful harvesting techniques and culture conditions that resulted in cell survival and propagation, and 3) how these cells performed in standard assessments of endothelial function. We hypothesised that this method would be feasible but not in all subjects and that the behaviour and function of these isolated cells could be further characterised. For this initial study, it was not our intent to link PAH disease state or subtype to PAEC phenotype.

\section{Methods}

Study sample

All patients referred to the Rhode Island Hospital Pulmonary Hypertension Center and undergoing RHC for the purposes of pulmonary vascular disease evaluation and management were eligible. We included all individuals undergoing RHC irrespective of their haemodynamics and clinical background in an effort to gain knowledge about patient-related and procedure-related characteristics more or less likely to result in successful PAEC harvest and sustained culture. Subjects with serial catheterisations during the study period were also included. The RHC procedure was not altered for the purposes of the study, nor was the number of times the catheter was manipulated or "wedged". All procedures were performed from the internal jugular position. Provocative manoeuvres during RHC, such as testing for vasoresponsiveness, exercise or fluid challenge, were left to the discretion of the treating pulmonary hypertension $(\mathrm{PH})$ clinician (CEV, CJM, JRK). In our centre we typically conduct vasoreactivity testing in all patients with suspected or confirmed pre-capillary PH on both index and follow-up RHC. The study was approved by the Lifespan Institutional Review Board (IRB \#016311) and informed consent was obtained from all participants. Medical records or our research registry were reviewed for clinical data, which was collected at the time of or as close as possible to RHC (i.e. within 6 months).

\section{Retention of PAC balloon tips and primary culture}

At the end of the RHC procedure, the PAC was retracted into the catheter sheath and both catheter and sheath were removed from the patient. The tip of the catheter was then advanced out of the sheath and placed directly into warm $\left(37^{\circ} \mathrm{C}\right.$ ) media (EndoGRO, Millipore Sigma, Billerica, MA, USA) with the balloon deflated. The rest of the PAC was then cut away and the tips in media were transported directly to the laboratory, initially at ambient temperature. Over the course of the study we noted that transport time and temperature change may have been factors in successful culture and began placing the warm media containing the balloon tip immediately into an 18 quart/12-volt powered compact warmer (Therapak Duramark Thermoelectric Warmer (part no. 365163), Claremont, CA, USA) custom preset to $37^{\circ} \mathrm{C}$ for travel to the laboratory. The PAC tip with balloon was placed directly into one well of a 24-well plate with attachment factor solution (Cell Applications Inc, San Diego, CA, USA) and media (EndoGRO-vascular endothelial growth factor (VEGF) complete media kit, Millipore Sigma, Billerica, MA, USA). The PAC tip was washed with fresh media every 2 days as cells attached to the cell culture dish and previous media from the tip well were moved into a new well in the 24-well plate. Cells were not treated with trypsin and were grown in primary culture to confluence over $2-3$ weeks. They were then seeded into T-25 and T-75 
flasks until passaged cells reached confluence over the next 4-5 days. After the third passage, cells were either directly characterised or frozen prior to further analysis.

Only the PH clinician conducting the RHC was aware of subject and procedural characteristics. Once the balloon tips were transferred to the laboratory, all study staff involved in further experiments (primary PAEC culture and the conduct of the characterisation assays) remained blinded to subject clinical data, RHC procedural details and haemodynamic values.

\section{Confirmation of PAEC phenotype and characterisation assays}

Primary PAECs obtained from catheter tips were screened for the expression of endothelial markers. Flow cytometry and fluorescence-activated cell sorting were utilised as a first step to confirm endothelial cell surface receptor pattern $\left(\mathrm{CD} 146^{+} / \mathrm{CD} 31^{+} / \mathrm{CD} 45^{-}\right.$) (data not shown). Once culture with cell confluence was achieved on recurrent PAC tip samples, we used a routine screening panel including von Willebrand factor (vWF) (Dako, Carpinteria, CA, USA) and vascular endothelial (VE)-cadherin (Santa Cruz Biotechnologies, Santa Cruz, CA, USA) staining, as well as acetylated low density lipoprotein (AcLDL) (ThermoFisher Scientific, Waltham, MA, USA) uptake to confirm PAEC phenotype. The cells were also stained for alpha-smooth muscle cell actin ( $\alpha$-SMA) (Abcam, Cambridge, MA, USA) as a negative control. Additional negative controls included staining human lung smooth muscle cells (Lonza, Pearland, TX, USA) and normal human lung fibroblasts (Lonza).

We considered cells to be endothelial in origin if all cells expressed all three endothelial markers (vWF, VE-cadherin staining and AcLDL uptake), were negative for $\alpha$-SMA and were able to generate Matrigel networks in vitro (as below). An attempt to culture PAECs from a balloon tip was considered successful if 1) cells grew to confluence, 2) cells remained viable in primary culture for at least 2-3 weeks, and 3) viable cells at 2-3 weeks exhibited an endothelial phenotype and expressed endothelial cell markers as described above and in the supplementary material. All studies of endothelial phenotype and function were done with PAECs between passages three to eight. Phenotypic drift, with subjective changes in PAEC morphology, was noted beyond passage nine via phase microscopy, immunofluorescence staining of endothelial cell markers and/or rates of proliferation. Technical replicates were performed such that each experiment included PAECs derived from a different passage of cells from a single balloon-tipped catheter from one RHC procedure for each subject.

Commercially available PAECs were purchased from Lonza Biologics Inc (Basel, Switzerland) or PromoCell (Heidelberg, Germany). These cells were maintained in the same manner as outgrown PAECs from PAC balloons and used as "normal" control PAECs for all experiments.

Standard terminal deoxynucleotidyl transferase dUTP nick end labeling (TUNEL), scratch migration and Matrigel tube formation assays were used to assess apoptosis, migration and tube formation, respectively [9-11]. Detailed methods are included in the supplementary material.

\section{Statistical analysis}

Continuous data are presented as median (interquartile range (IQR)) and categorical data are presented as n (\%). Wilcoxon-Mann-Whitney tests were used to compare continuous variables and Chi-squared or Fisher exact tests were used to compare categorical variables for successful versus unsuccessful attempts, as appropriate. Characterisation assays were compared by two-way ANOVA with correction for multiple comparisons. All hypotheses were tested using two-tailed tests. Alpha was established at the 0.05 level.

\section{Results}

Subject and procedural factors and culture success

A total of 49 PAC tips were collected from 45 subjects with successful culture of PAECs from 19 balloons (39\%) using our current protocol. Table 1 describes subject and procedural characteristics by PAEC culture success. Subject demographics and anthropometrics were similar in successful versus unsuccessful attempts. There were no differences in the number of clinical PAH diagnoses (i.e. previous World Health Organization (WHO) Group $1 \mathrm{PAH}$ designation by a $\mathrm{PH}$ specialist), WHO functional class, or targeted PAH therapy use at the time of catheterisation. Shorter 6-min walk distance (6MWD) may have been associated with culture success in successful (355 m, IQR $122-410 \mathrm{~m})$ versus unsuccessful $(375 \mathrm{~m}$, IQR 295-462 m) attempts $(\mathrm{p}=0.09)$. There was a higher proportion of classical haemodynamic PAH (i.e. mean pulmonary arterial pressure (PAP) $\geqslant 25 \mathrm{mmHg}$, mean pulmonary capillary wedge pressure (PCWP) $\leqslant 15 \mathrm{mmHg}$ and pulmonary vascular resistance (PVR) $>3$ Wood units) among successful attempts (10 out of 19,53\%) versus unsuccessful attempts (nine out of 30,30\%), although this difference was not statistically significant $(p=0.10)$. Results were unchanged when the mean PAP cutoff was lowered to $20 \mathrm{mmHg}$ [12]. Successful culture of PAECs from a balloon-tipped PAC was more likely in subjects who had more severe haemodynamic impairment at the time of RHC, as evidenced by significantly lower 


\begin{tabular}{|c|c|c|c|c|}
\hline & Total sample & Success & No success & p-value \\
\hline Balloon tips & 49 (100) & 19 (39) & $30(61)$ & \\
\hline \multicolumn{5}{|l|}{ Subject-level characteristics } \\
\hline Age years & $62(53-71)$ & $69(54-78)$ & $61(52-67)$ & 0.16 \\
\hline Male sex & $17(35)$ & $8(42)$ & $9(30)$ & 0.29 \\
\hline Race/Ethnicity & & & & 0.86 \\
\hline White & 34 (69) & $13(68)$ & $21(70)$ & \\
\hline Black & $11(22)$ & $4(21)$ & $7(23)$ & \\
\hline Other & $3(6)$ & $2(11)$ & $1(3)$ & \\
\hline $\mathrm{BMI} \mathrm{kg} \cdot \mathrm{m}^{-2}$ & $30(25-33)$ & $32(27-34)$ & $29(24-33)$ & 0.25 \\
\hline Clinical PAH diagnosis & $22(45)$ & $8(42)$ & $14(47)$ & 0.49 \\
\hline PAH treatment & 19 (39) & $6(32)$ & $13(43)$ & 0.30 \\
\hline 6MWD m & $365(182-425)$ & $355(122-410)$ & $375(295-462)$ & 0.09 \\
\hline Functional class III/IV & $15(47)$ & $6(46)$ & $9(47)$ & 1.00 \\
\hline Number available & 32 & 13 & 19 & \\
\hline $\mathrm{BNP} \mathrm{pg} \cdot \mathrm{mL}^{-1}$ & $80(23-390)$ & $76(32-413)$ & $80(22-184)$ & 0.71 \\
\hline Number available & 42 & 26 & 16 & \\
\hline Haemodynamic PH & $39(80)$ & $16(84)$ & $23(77)$ & 0.40 \\
\hline Haemodynamic PAH & 19 (39) & $10(53)$ & $9(30)$ & 0.10 \\
\hline \multicolumn{5}{|l|}{ Haemodynamics } \\
\hline RAP mmHg & $9(7-12)$ & $10(7-14)$ & $9(6-11)$ & 0.40 \\
\hline mean PAP mmHg & $37(25-46)$ & $38(26-48)$ & $36(25-42)$ & 0.34 \\
\hline PCWP mmHg & $12(10-16)$ & $13(9-15)$ & $12(10-16)$ & 0.77 \\
\hline $\mathrm{COL} \cdot \mathrm{min}^{-1}$ & $5.5(4.4-7.7)$ & $4.7(4.0-5.6)$ & $6.4(4.7-8.1)$ & 0.04 \\
\hline Cardiac index $\mathrm{L} \cdot \mathrm{min}^{-1} \cdot \mathrm{m}^{-2}$ & $2.3(2.1-3.5)$ & $2.3(2.1-3.5)$ & $3.2(2.8-4.0)$ & 0.03 \\
\hline PVR Wood units & $3.6(1.9-6.5)$ & $5.1(2.2-8.3)$ & $2.4(1.5-6.2)$ & 0.04 \\
\hline \multicolumn{5}{|l|}{ Procedural-level characteristics } \\
\hline Provocative testing ${ }^{\#}$ & $33(67)$ & $14(74)$ & $19(63)$ & 0.64 \\
\hline Most experienced operator & 19 (39) & 7 (37) & $12(40)$ & 0.77 \\
\hline Morning collection" & $33(67)$ & $14(74)$ & $19(63)$ & 0.54 \\
\hline Transit time ${ }^{+} \min$ & $28(20-40)$ & $28(25-40)$ & $28(19-49)$ & 0.77 \\
\hline \multicolumn{5}{|l|}{ Transport conditions } \\
\hline Media $37^{\circ} \mathrm{C}$ (no heater) & $43(88)$ & $15(35)$ & $28(65)$ & 0.19 \\
\hline Media $37^{\circ} \mathrm{C}$ (heater $37^{\circ} \mathrm{C}$ ) & $6(12)$ & $4(67)$ & $2(33)$ & \\
\hline
\end{tabular}

Data are presented as $\mathrm{n}(\%)$ or median (IQR) unless otherwise stated. Wilcoxon-Mann-Whitney tests were used to compare continuous variables and Chi-squared or Fisher exact tests were used to compare categorical variables, as appropriate. Haemodynamic values were taken from baseline assessment. All catheterisations were performed from the internal jugular position. BMI: body mass index; PAH: pulmonary arterial hypertension (defined as mean PAP $\geqslant 25 \mathrm{mmHg}$, PCWP $\leqslant 15 \mathrm{mmHg}$ and PVR $>3$ Wood units); 6MWD: 6-min walk distance; BNP: brain natriuretic peptide; $\mathrm{PH}$ : pulmonary hypertension (defined as mean $P A P \geqslant 25 \mathrm{mmHg}$ ); RAP: right-atrial pressure; PAP: pulmonary arterial pressure; PCWP: pulmonary capillary wedge pressure; CO: cardiac output; PVR: pulmonary vascular resistance. \#: manoeuvres such as vasoreactivity or exercise testing requiring more than one "wedging" of the catheter; ${ }^{\Uparrow}$ : pulmonary artery catheter tip collected before $12 \mathrm{pm} ;{ }^{+}$: Ex vivo to culture dish.

cardiac output $(\mathrm{CO})(\mathrm{p}=0.04)$ and cardiac index $(\mathrm{p}=0.03)$, as well as higher PVR $(\mathrm{p}=0.04)$. Procedural details such as the number of times the PAC was "wedged", the specific operator (i.e. the clinician with the most experience performing RHC) and the transit time ex vivo to the culture dish did not influence success. We used a heater for transport of six balloons toward the end of the study, which may have increased the success rate $(\mathrm{p}=0.19)$.

Detailed characteristics of the subjects with PAEC culture success are provided in table 2. Three subjects had more than one RHC during the study period. Subject 7 in table 2 (a 56-year old woman with portopulmonary hypertension $(\mathrm{PoPH})$ ) had three catheterisations, one of which yielded successfully cultured PAECs. We cultured PAECs from only one of two PAC tips from subject 10 (a 58-year old man with HIV infection and chronic obstructive pulmonary disease (COPD)). In both cases there were no changes to the study protocol between procedures and PAH medications as well as haemodynamics were unchanged and similar, respectively, across procedures. Results were unchanged when these subjects were excluded from analyses. A third subject with systemic sclerosis associated-PAH (data not shown) had two RHCs but we were unsuccessful in culturing PAECs from both balloons. 


\begin{tabular}{|c|c|c|c|c|c|c|c|c|c|}
\hline Subject & Age & Sex & Diagnosis & $\begin{array}{c}\text { RAP } \\
\mathrm{mmHg}\end{array}$ & $\begin{array}{l}\text { Mean } \\
\text { PAP } \\
\text { mmHg }\end{array}$ & $\begin{array}{l}\text { PCWP } \\
\mathrm{mmHg}\end{array}$ & $\frac{\mathrm{CO}}{\mathrm{L} \cdot \mathrm{min}^{-1}}$ & $\begin{array}{l}\text { PVR } \\
\text { Wood } \\
\text { units }\end{array}$ & $\begin{array}{l}\text { PAH } \\
\text { time }\end{array}$ \\
\hline 1 & 75 & $\mathrm{~F}$ & PAPVR & 12 & 26 & 14 & 9.9 & 1.2 & ERA \\
\hline 2 & 70 & M & Systemic sclerosis & 17 & 47 & 8 & 4.7 & 8.3 & None \\
\hline 3 & 79 & $\mathrm{~F}$ & $\mathrm{CPC}-\mathrm{PH}$ & 13 & 41 & 22 & 3.8 & 5.0 & None \\
\hline 4 & 78 & M & $\begin{array}{l}\text { Anti-synthetase } \\
\text { syndrome, ILD }\end{array}$ & 14 & 53 & 15 & 3.9 & 9.7 & None \\
\hline 5 & 37 & $\mathrm{~F}$ & $\begin{array}{c}\text { IPAH, } \\
\text { vasoresponder }\end{array}$ & 7 & 25 & 9 & 7.4 & 2.2 & $\begin{array}{l}\text { PDE5i, } \\
\text { ERA, } \\
\text { CCB }\end{array}$ \\
\hline 6 & 69 & M & $\begin{array}{c}\text { Congenital heart } \\
\text { disease }\end{array}$ & 18 & 48 & 16 & 5.1 & 6.3 & PDE5 \\
\hline $7^{+}$ & 56 & $\mathrm{~F}$ & $\mathrm{PoPH}$ & 4 & 34 & 12 & 12.9 & 1.7 & $\begin{array}{l}\text { PDE5i, } \\
\text { ERA, PA }\end{array}$ \\
\hline 8 & 84 & $\mathrm{~F}$ & $\begin{array}{c}\text { Systemic } \\
\text { sclerosis, HFpEF }\end{array}$ & 15 & 60 & 22 & 3.3 & 11.5 & None \\
\hline 9 & 59 & $\mathrm{~F}$ & $\begin{array}{c}\text { Systemic } \\
\text { sclerosis, exercise } \\
\text { PH }\end{array}$ & 10 & 21 & 13 & 7.7 & 1.0 & None \\
\hline $10^{+}$ & 58 & M & HIV, COPD & 10 & 29 & 16 & 5.0 & 2.6 & ERA \\
\hline 11 & 44 & M & Sarcoid & 24 & 50 & 6 & 4.5 & 9.8 & None \\
\hline 12 & 84 & $\mathrm{~F}$ & IPAH & 10 & 43 & 13 & 4.5 & 6.7 & None \\
\hline 13 & 52 & $\mathrm{~F}$ & HIV & 14 & 57 & 11 & 4.9 & 9.4 & $\begin{array}{l}\text { PDE5i, } \\
\text { PA }\end{array}$ \\
\hline 14 & 72 & M & $\begin{array}{l}\text { Unexplained } \\
\text { dyspnoea }\end{array}$ & 7 & 20 & 11 & 4.1 & 2.2 & None \\
\hline 15 & 81 & $\mathrm{~F}$ & $\begin{array}{c}\text { COPD, HFpEF, } \\
\text { OSA }\end{array}$ & 8 & 35 & 13 & 4.3 & 5.1 & None \\
\hline 16 & 68 & $\mathrm{~F}$ & $\begin{array}{l}\text { Sjogren's } \\
\text { syndrome }\end{array}$ & 7 & 24 & 9 & 3.8 & 3.9 & None \\
\hline 17 & 54 & M & ESRD, OSA, ILD & 8 & 46 & 15 & 4.8 & 6.5 & None \\
\hline 18 & 43 & $\mathrm{~F}$ & IPAH & 4 & 33 & 6 & 5.6 & 4.8 & $\begin{array}{l}\text { PDE5i, } \\
\text { ERA }\end{array}$ \\
\hline 19 & 76 & M & COPD & 2 & 38 & 7 & 3.9 & 7.9 & None \\
\hline
\end{tabular}

RAP: right-atrial pressure; PAP: pulmonary arterial pressure; PCWP: pulmonary capillary wedge pressure; CO: cardiac output; PVR: pulmonary vascular resistance; PAH: pulmonary arterial hypertension; PAPVR partial anomalous pulmonary venous return; CPC-PH: combined pre- and post-capillary pulmonary hypertension; ILD: interstitial lung disease; IPAH: idiopathic PAH; PoPH: portopulmonary hypertension; HFpEF: heart failure with preserved ejection fraction; $\mathrm{PH}$ : pulmonary hypertension; COPD: chronic obstructive pulmonary disease; OSA: obstructive sleep apnoea; ESRD: end-stage renal disease; PDE5i: phosphodiesterase type-5 inhibitor; ERA: endothelin receptor antagonist; CCB: calcium channel blocker; PA: prostacyclin analogue. \#: PAH treatment (time of procedure); ${ }^{+}$: subject 7: culture success from one out of three balloons; subject 10: culture success from one out of two balloons.

\section{PAEC characterisation}

Cells demonstrated marked variability in the ability and speed at which they grew to confluence. Outgrowth of cells from the balloon tip could be observed within the first several days in culture, but confluence in the 24-well plate varied from 6 days to 4 weeks. In early experiments, we performed manual cell counts using trypan blue (ThermoFisher Scientific, Waltham, MA, USA) and these ranged from 12500 to 968000 . Routine cell counts were not part of our protocol for the data presented herein. We confirmed PAEC phenotype in primary culture and during subsequent passages and then, when yield allowed, conducted characterisation assays. Figure 1 and table 3 demonstrate confirmation of PAEC phenotype in both primary and passaged cells on representative samples for the following subjects (selected because of sufficient yield and because they illustrated a range of clinical and cellular phenotypes): 1) a man (subject 14 in table 2) with a mean $\mathrm{PAP}=20 \mathrm{mmHg}$, referred for unexplained dyspnoea but without documented cardiopulmonary disease, whose cells had slow proliferation qualitatively; 2) a woman (subject 18 in table 2) with idiopathic PAH (IPAH), on a phosphodiesterase type-5 inhibitor (PDE5i) and an endothelin receptor antagonist (ERA), who had improvement in pulmonary haemodynamics in response to these medications and a moderately elevated PVR; 3 ) a woman 

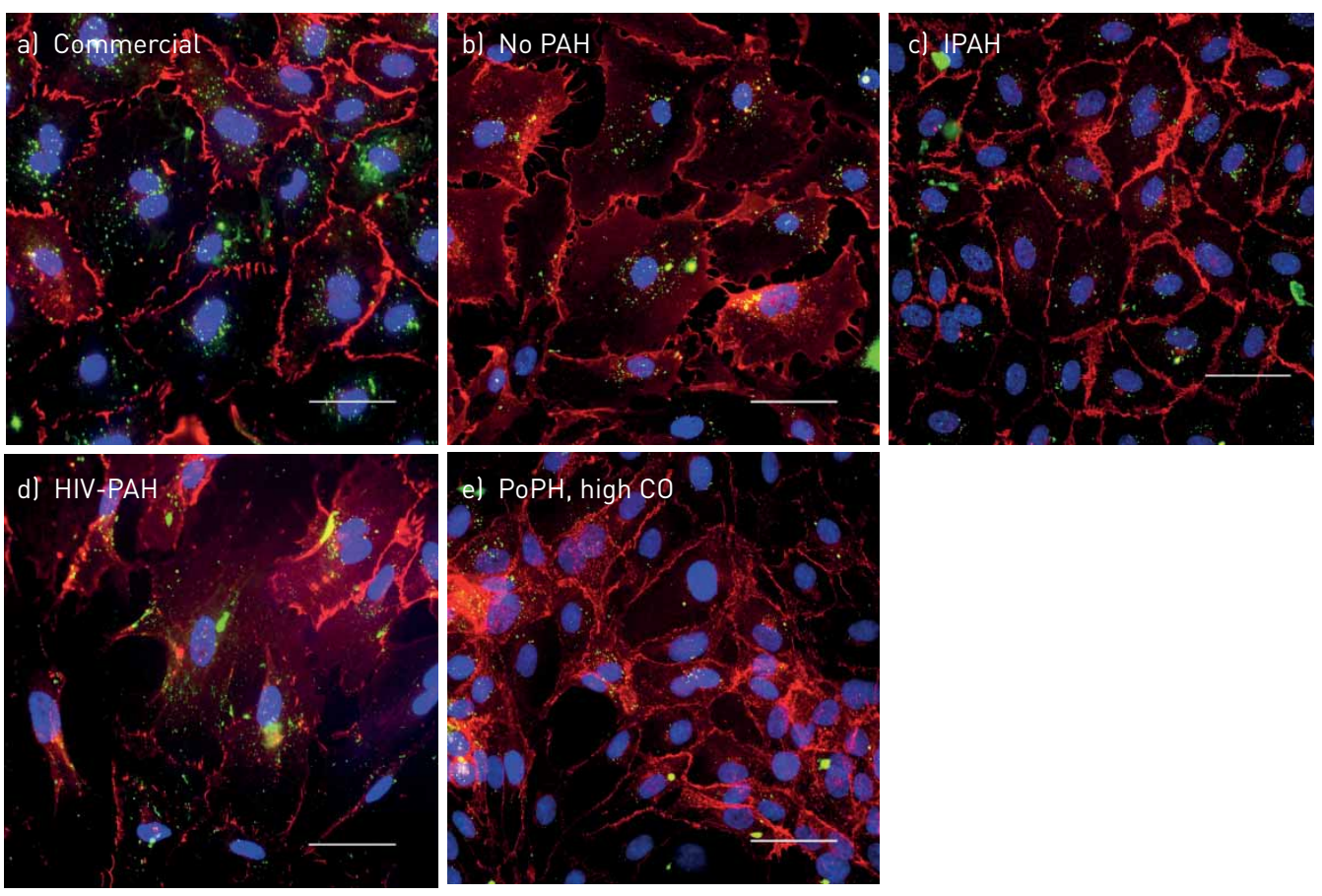

FIGURE 1 Representative images confirming endothelial cell phenotypes, as follows: (a) commercial human PAECs (passage six); (b) no pulmonary arterial hypertension (PAH) (subject 14, passage four); (c) idiopathic PAH (IPAH) (subject 18, passage three); (d) HIV-associated PAH (HIV-PAH) (subject 13, passage four); (e) portopulmonary hypertension (PoPH) with high cardiac output (CO) (subject 7, passage four). In all images, VE-cadherin staining is red and AcLDL uptake is green (magnification=40x; scale bars=50 $\mu \mathrm{m}$ ).

(subject 13 in table 2) with HIV-associated PAH (HIV-PAH), on a PDE5i and prostacyclin analogue (PA), with severe haemodynamic compromise and high PVR; and 4) a woman (subject 7 in table 2) with PoPH, on triple PAH therapy (PA, PDE5i and ERA) with a high-output, low PVR state at the time of cardiac catheterization, whose cells were rapidly proliferative. As noted by the immunofluorescence staining in figure 1 and table 3, cells stained positive for VE-cadherin and vWF, with uptake of AcLDL and were negative for $\alpha$-SMA. To confirm the specificity of the endothelial cell markers used, further testing demonstrated primary cultures of human lung smooth muscle cells and lung fibroblasts did not stain for VE-cadherin, CD31 or vWF, nor did they take up AcLDL (see supplementary material, supplementary table E1 and supplementary figure E1).

\section{Apoptosis in PAH PAECs}

Treatment with tumour necrosis factor- $\alpha$ (TNF- $\alpha$ ) induced apoptosis detected via TUNEL assay in commercial PAECs, in cells from the subject without PAH (subject 14) and, to a lesser extent, in cells from the subject with PoPH and high CO (subject 7) (figure 2). PAECs from the subject with IPAH (subject 18) were highly resistant to apoptosis in response to TNF- $\alpha$ treatment and had significantly lower rates of apoptosis than commercial PAECs ( $\mathrm{p}=0.042$, corrected for multiple comparisons). We were

\section{TABLE 3 Confirmation of PAEC phenotype from representative samples}

\begin{tabular}{lcccccc} 
Subject & Details & CD-31 & vWF & VE-cadherin & AcLDL uptake & $\boldsymbol{\alpha}$-SMA \\
\hline 14 & No PH & + & + & + & + & - \\
18 & IPAH, controlled haemodynamics & + & + & + & + & + \\
13 & HIV-PAH, high PVR & + & + & + & + & - \\
7 & PoPH, high CO, low PVR & + & + & + & + & -
\end{tabular}

+ indicates that $100 \%$ of cells stained positive for antibody or took up AcLDL; - indicates that all cells were negative for antibody staining. vWF: von Willebrand factor; VE: vascular endothelial; AcLDL: acetylated low density lipoprotein; $\alpha$-SMA: alpha-smooth muscle cell actin; PAH: pulmonary arterial hypertension; IPAH: idiopathic PAH; HIV-PAH: HIV-associated PAH; PVR: pulmonary vascular resistance; PoPH: portopulmonary hypertension; $\mathrm{CO}$ : cardiac output. 

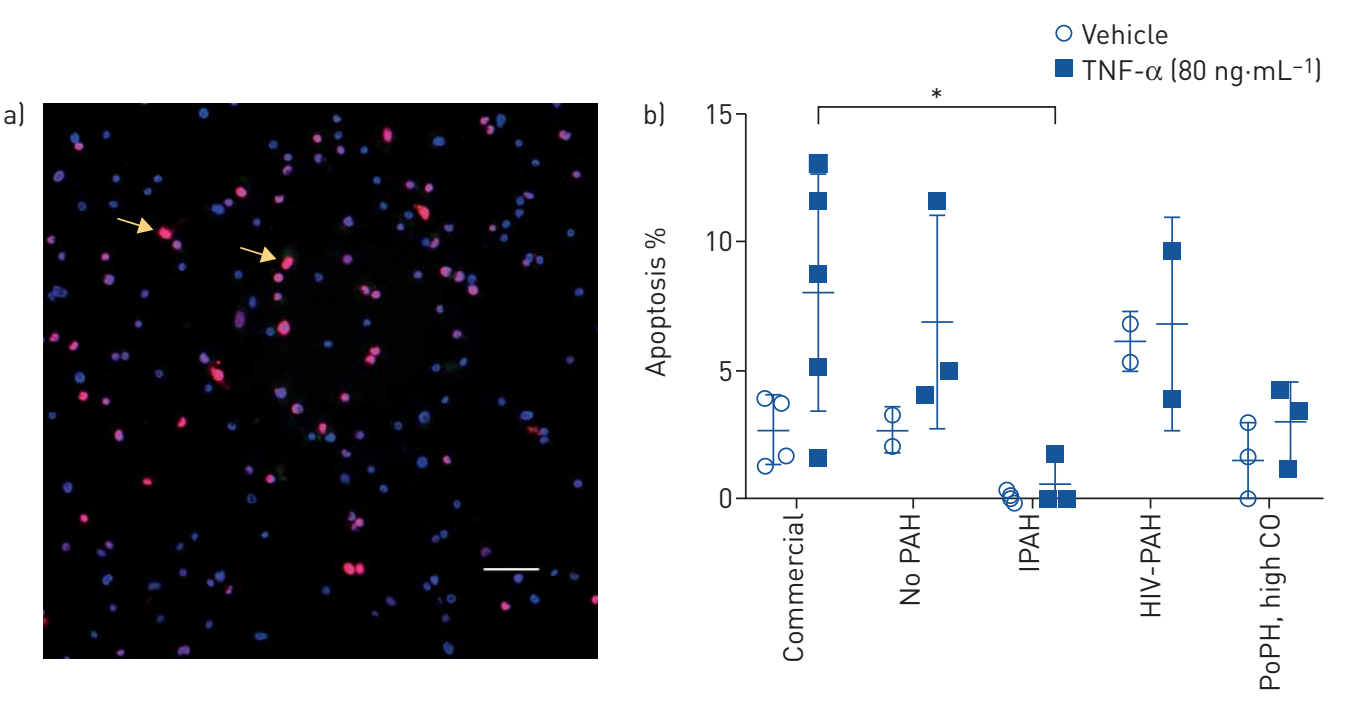

FIGURE 2 Apoptosis assessed by terminal deoxynucleotidyl transferase dUTP nick end labeling (TUNEL) assay following treatment with vehicle and tumour necrosis factor- $\alpha$ (TNF- $\alpha$ ). Panels are as follows: (a) representative image of PAECs exposed to tert-butyl hydroperoxide for $6 \mathrm{~h}$, TUNEL stained for apoptosis and counterstained with 4',6-diamidino-2-phenylindole (DAPI). TUNEL-positive cells are indicated by yellow arrows (magnification=20x; scale bar=50 $\mu \mathrm{m}$ ); (b) degree of apoptosis when PAECs were exposed to vehicle or TNF- $\alpha$ for $6 \mathrm{~h}$. Data are presented as mean \pm SD. $n=2-5$ and each $n$ is derived from a different passage of cells from a single balloon-tipped catheter from one $\mathrm{RHC}$ procedure for a single subject (no $\mathrm{PAH}=$ subject 14; IPAH=subject 18; HIV-PAH=subject 13; $\mathrm{PoPH}$, high $\mathrm{CO}=$ subject 7). $\mathrm{PAH}$ : pulmonary arterial hypertension; IPAH: idiopathic PAH; HIV-PAH: HIV-associated PAH; PoPH: portopulmonary hypertension; CO: cardiac output. $*$ : $p<0.05$.

limited in our ability to characterise apoptosis in PAECs from the subject with HIV-PAH and high PVR (subject 13), due to a low yield of cells $(n=2)$; however, apoptotic response trends in cells from this subject were similar to those in commercial PAECs.

\section{Migration and tube formation by PAH phenotype}

We successfully characterised migration in PAECs from three of the four subjects using a standard scratch migration assay (figure 3). The subject with $\mathrm{PoPH}$ and high $\mathrm{CO}$ (subject 7) had higher rates of migration as compared to the subject with IPAH (subject 18) in low serum ( $p=0.039$, corrected for multiple comparisons) and in response to complete medium ( $\mathrm{p}=0.006$, corrected for multiple comparisons) (figure 3 ). The rate of migration in low serum was also higher in the subject with PoPH as compared to the subject with no PAH (subject 14) ( $\mathrm{p}=0.025)$. We were able to fully assess tube formation by Matrigel assay in PAECs from all four subjects. Cells from the subject with HIV-PAH and high PVR (subject 13) were less likely to form branching vessels ( $\mathrm{p}=0.014$, corrected for multiple comparisons) and branching vessels were shorter in length ( $\mathrm{p}=0.022$, corrected for multiple comparisons) as compared to commercial PAECs exposed to VEGF (figure 4). There were no significant differences in mesh size, number or length of segments, or number of mesh rings (data not shown) across PAECs from subjects as compared to commercial PAECs.

\section{Discussion}

We have demonstrated that PAECs can be harvested and sustained in culture from PAC balloon tips used during routine haemodynamic evaluation. Primary cells were maintained in culture out to 4 weeks and were confirmed to have endothelial cell phenotype and to express endothelial cell markers through several passages. Cells isolated by this technique demonstrated functional characteristics that were generally similar to commercially available human PAECs. Our findings suggest that this approach could be harnessed and further developed to identify and characterise abnormalities in endothelial cell function in patients with pulmonary vascular disease.

The ability to successfully culture PAECs and variability in the harvested cell phenotype may be related to an individual's genetic background, treatment, environmental exposures, epigenetic and pharmacogenomic changes, and/or the procedure itself. The earlier report by Pollett et al [8] commented that successful culture was influenced by the clinician performing the RHC and the hydration status of the patient, but did not quantitate these findings. We found that our success rate was not associated with operator experience or most subject characteristics. Protocol details for PAC-tip processing and PAEC harvest differed substantially 

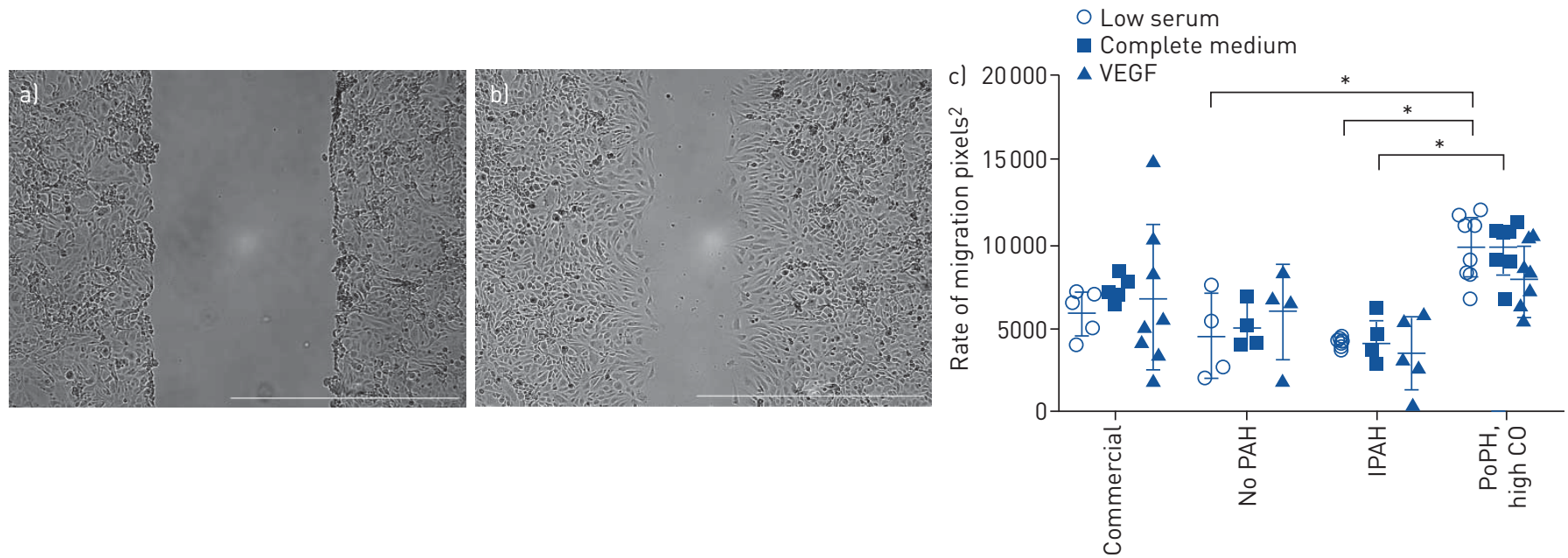

FIGURE 3 Migration assessed by scratch assay under low serum conditions, complete medium conditions and low serum conditions with vascular endothelial growth factor (VEGF). Representative phase images of PAECs are shown at $0 \mathrm{~h}(\mathrm{a})$ and $6 \mathrm{~h}$ (b) after scratch (magnification=4x; scale bar $=100 \mu \mathrm{m}$ ). Rate of migration is shown in panel (c). Data are presented as mean $\pm S D . n=3-8$ and each $n$ is derived from a different passage of

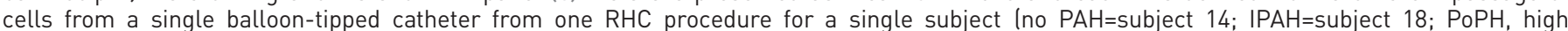
$\mathrm{CO}=$ subject 7]. PAH: pulmonary arterial hypertension; IPAH: idiopathic $\mathrm{PAH} ; \mathrm{PoPH}$ : portopulmonary hypertension; $\mathrm{CO}$ : cardiac output. *: $\mathrm{p}<0.05$.

a)


FIGURE 4 Tube formation following Matrigel treatment as quantified by AngioTool. Representative phase images of tube formation (a) are shown at $2 \mathrm{~h}$ and $6 \mathrm{~h}$ following the seeding of PAECs on Matrigel (magnification=4x; scale bars $=100 \mu \mathrm{m}$ ). Further data are shown graphically, including mesh size (b), number of segments (c), number of branches (d), segment length (e) and branch length ( $f$ ). Data are presented as mean $\pm 5 D$. $n=4-8$ and each $\mathrm{n}$ is derived from a different passage of cells from a single balloon-tipped catheter from one RHC procedure for a single subject (no $\mathrm{PAH}=$ subject 14; IPAH=subject 18; HIV-PAH=subject 13; $\mathrm{PoPH}$, high $\mathrm{CO}=$ subject 7). $\mathrm{PAH}$ : pulmonary arterial hypertension; IPAH: idiopathic PAH; HIV-PAH: HIV-associated PAH; PoPH: portopulmonary hypertension; CO: cardiac output. *: $p<0.05$. 
from the prior report (e.g.we did not include trypsinisation, centrifugation, lysis or cell sorting steps) which may have increased our yield. We did observe that the degree of haemodynamic compromise contributed to the success of PAEC culture. We were more likely to obtain viable PAECs from PAH patients with more severe disease (as evidenced by haemodynamics and possibly shorter 6MWD), which in part may be related to our observation that PAH PAECs were more apoptosis resistant compared to commercial PAECs. Altered mechanical properties (i.e. hyperpermeability and/or altered motility) of PAH PAECs may have led to increased transfer from the endothelium to the PAC balloon, as has been shown in experimental $\mathrm{PH}$ [13]. However, these observations are preliminary and require confirmation.

Distal PAECs from explanted PAH lungs have increased proliferation and decreased apoptosis as compared to control lung PAECs [14]. This mirrors our in vitro qualitative and (to a limited extent) quantitative observations, in which cells from subjects with PAH and higher PVR tended to be rapidly proliferative and apoptosis resistant. Others have shown that PAH is characterised by accelerated endothelial cell apoptosis with loss of vasculature and impaired migration [15]. While we demonstrated some differences in migration and network formation depending on PAH subtype, whether or not these patterns are typical will require a larger number of subjects.

Functional abnormalities of PAECs from a second-order or third-order pulmonary artery may not represent those from the distal resistance vessels implicated in PAH. In healthy rats, lung microvascular endothelial cells have higher proliferative potential than proximal PAECs, but also significant microheterogeneity in replication competency that depends on the parent cell population [16]. It is possible that similar microheterogeneity exists in human proximal PAECs (or local progenitors) and that single cell cloning could reduce the variability we observed. There is also a rationale for studying proximal PAECs, given that wall stress changes in proximal pulmonary arteries contribute to compliance and coupling in PAH [17].

This preliminary report has limitations. Cultured endothelial cells could have derived from the central veins or right heart, as well as from circulating blood outgrowth endothelial cells (BOECs). Only the balloon and catheter tip are collected which contains $17-33$-fold less blood (at most $2.4 \mathrm{~mL}$ and typically $<1 \mathrm{~mL}$ ) than the $40-80 \mathrm{~mL}$ required for isolation of BOECs [7]. It is unlikely that sufficient numbers of endothelial cells from elsewhere could attach to the balloon during incidental contact en route to the wedge position. No distinct "omic" signature is able to differentiate PAECs from other locations and we contend that the source of cells is most likely the pulmonary artery where the balloon has by far the greatest surface-to-surface contact. As a proof-of-concept, in two subjects who recently underwent RHC we advanced a PAC to the right ventricle, but no further, and did not inflate the balloon. Neither of these PAC tips yielded endothelial cells. In both procedures, a second catheter was then used to float to the pulmonary artery and then into the wedge position, with the balloon inflated to complete the procedure. In the patient with PAH (the second had PH/pulmonary venous hypertension), we successfully cultured PAECs from this catheter tip. The experiments conducted represent technical, not biological, replicates. We were unable to culture cells from serial catheterisations of several subjects performed during the study period despite the same protocol and, in some cases, cell yield limited the number of experiments for a given assay (e.g. characterisation of apoptosis in HIV-PAH). After we introduced the heater for sample transport, the subject with PoPH (subject 7) had three subsequent catheterisations, from which all three balloons yielded PAECs (a total within-subject success rate of four out of six (67\%). Characterisation of PAEC behaviour in these biological replicates and banked PAECs from all successful attempts is the subject of ongoing work. Finally, we do not know the bone morphogenetic receptor type-2 (BMPR2) mutation status of the subjects, although none of the participants had heritable disease. PAECs from patients with BMPR2 mutations may be pro-proliferative as compared to other forms of $\mathrm{PAH}$ and may have contributed to our higher success rates in patients with more severe PAH [18]. A larger sample size is needed to draw conclusions about the functional differences seen here. It is our goal to publish these early observations so that the rigour of the method can be improved as a necessary first step. Finally, we were encouraged that we had greater success culturing PAECs from subjects with the disease of interest (i.e. haemodynamic PAH, higher PVR states) in which this technique may prove the most scientifically fruitful.

\section{Conclusions}

Routine RHC for pulmonary vascular disease evaluation may represent a novel opportunity for successful harvest and culture of PAECs from PAC balloon tips, especially in individuals with greater haemodynamic compromise.

Conflict of interest: C.E. Ventetuolo reports grants from National Institutes of Health (R01 HL141268 and P20GM103652), during the conduct of the study; institutional grants from United Therapeutics and Eiger; personal fees from Acceleron Pharma, outside the submitted work; and her spouse was a recent employee of CVS Health. J.M. Aliotta has nothing to disclose. J. Braza has nothing to disclose. H. Chichger has nothing to disclose. M. Dooner has nothing to 
disclose. D. McGuirl has nothing to disclose. C.J. Mullin has nothing to disclose. J. Newton has nothing to disclose. M. Pereira has nothing to disclose. A. Princiotto has nothing to disclose. P.J. Quesenberry has nothing to disclose. T. Walsh has nothing to disclose. M. Whittenhall has nothing to disclose. J.R. Klinger has nothing to disclose. E.O. Harrington has nothing to disclose.

Support statement: This work was completed with support from an Institutional Development Award (IDeA) from the National Institute of General Medical Sciences (P20 GM103652) and a National Institutes of Health grant (R01-HL141268). Funding information for this article has been deposited with the Crossref Funder Registry.

\section{References}

1 Budhiraja R, Tuder RM, Hassoun PM. Endothelial dysfunction in pulmonary hypertension. Circulation 2004; 109: 159-165.

2 Morrell NW, Adnot S, Archer SL, et al. Cellular and molecular basis of pulmonary arterial hypertension. J Am Coll Cardiol 2009; 54: S20-S31.

3 Tuder RM, Archer SL, Dorfmuller P, et al. Relevant issues in the pathology and pathobiology of pulmonary hypertension. J Am Coll Cardiol 2013; 62: D4-D12.

4 Humbert M, Guignabert C, Bonnet S, et al. Pathology and pathobiology of pulmonary hypertension: state of the art and research perspectives. Eur Respir J 2019; 53: 1801887.

5 Gu M, Shao NY, Sa S, et al. Patient-specific iPSC-derived endothelial cells uncover pathways that protect against pulmonary hypertension in BMPR2 mutation carriers. Cell Stem Cell 2017; 20: 490-504.

6 Sa S, Gu M, Chappell J, et al. Induced pluripotent stem cell model of pulmonary arterial hypertension reveals novel gene expression and patient specificity. Am J Respir Crit Care Med 2017; 195: 930-941.

7 Geti I, Ormiston ML, Rouhani F, et al. A practical and efficient cellular substrate for the generation of induced pluripotent stem cells from adults: blood-derived endothelial progenitor cells. Stem Cells Transl Med 2012; 1: 855-865.

8 Pollett JB, Benza RL, Murali S, et al. Harvest of pulmonary artery endothelial cells from patients undergoing right heart catheterization. J Heart Lung Transplant 2013; 32: 746-749.

9 Lu Q, Patel B, Harrington EO, et al. Transforming growth factor- $\beta 1$ causes pulmonary microvascular endothelial cell apoptosis via ALK5. Am J Physiol Lung Cell Mol Physiol 2009; 296: L825-L838.

10 Ottosson M, Jakobsson A, Johansson F. Accelerated wound closure - differently organized nanofibers affect cell migration and hence the closure of artificial wounds in a cell based in vitro model. PLoS One 2017; 12: e0169419.

11 Zudaire E, Gambardella L, Kurcz C, et al. A computational tool for quantitative analysis of vascular networks. PLoS One 2011; 6: e27385.

12 Simonneau G, Montani D, Celermajer DS, et al. Haemodynamic definitions and updated clinical classification of pulmonary hypertension. Eur Respir J 2019; 53: 1801913.

13 Zhou C, Francis CM, Xu N, et al. The role of endothelial leak in pulmonary hypertension (2017 Grover Conference Series). Pulm Circ 2018; 8: 2045894018798569.

14 Masri FA, Xu W, Comhair SA, et al. Hyperproliferative apoptosis-resistant endothelial cells in idiopathic pulmonary arterial hypertension. Am J Physiol Lung Cell Mol Physiol 2007; 293: L548-L554.

15 Nickel NP, Spiekerkoetter E, Gu M, et al. Elafin reverses pulmonary hypertension via caveolin-1-dependent bone morphogenetic protein signaling. Am J Respir Crit Care Med 2015; 191: 1273-1286.

16 Lee JY, McMurtry SA, Stevens T. Single cell cloning generates lung endothelial colonies with conserved growth, angiogenic, and bioenergetic characteristics. Pulm Circ 2017; 7: 777-792.

17 Schafer M, Kheyfets VO, Schroeder JD, et al. Main pulmonary arterial wall shear stress correlates with invasive hemodynamics and stiffness in pulmonary hypertension. Pulm Circ 2016; 6: 37-45.

18 Ferrer E, Dunmore BJ, Hassan D, et al. A potential role for exosomal translationally controlled tumor protein export in vascular remodeling in pulmonary arterial hypertension. Am J Respir Cell Mol Biol 2018; 59: 467-478. 